\title{
Nepal Paediatric Society Guidelines for the Safe Transport of Critically Ill Children in Nepal
}

\section{Puja Amatya ${ }^{1}$, Dhruba Shrestha ${ }^{2}$, Suchita Joshi ${ }^{3}$, Arun Sharma ${ }^{4}$, Shrijana Shrestha and Sangita Basnet ${ }^{6}$}

${ }^{1}$ Department of Paediatrics, Patan Academy of Health Sciences, Lalitpur, Nepal

${ }^{2}$ Department of Paediatrics, Siddhi Memorial Hospital, Bhaktapur, Nepal

${ }^{3}$ Department of Paediatrics, Nepal Mediciti Hospital, Lalitpur, Nepal

${ }^{4}$ Department of Paediatrics, IOM, Kathmandu and Organising Secretary NEPAS, Nepal

${ }^{5}$ Department of Paediatrics and Dean of Patan Academy of Health Sciences, Lalitpur, Nepal

${ }^{6}$ Department of Paediatrics, SIU School of Medicine, USA

\section{ABSTRACT}

Transport of critically ill children is associated with increased risk of morbidity and mortality in developing countries due to the lack of adequate resources and skilled personnel. Transport of the sick child remains a major challenge in Nepal as well. The main objective of this article is to describe the importance of safe transport, and be a resource and guide for health care personnel during inter-facility transport of sick children in Nepal. This guideline is based on consensus statement of Nepal Paediatric Critical Care Working Group (NPCCWG) under the aegis of the Nepal Paediatric Society (NEPAS). It includes different components of transport, namely essential equipment, patient preparation, communications, medications, and transport checklist, that are required during transport of sick children, taking into consideration the current resources available in our health care facilities.

Key words: Critically ill child; Nepal Paediatric Critical Care Working Group (NPCCWG); Nepal Paediatric Society (NEPAS); Paediatric Intensive Care Unit (PICU); Transport

\section{Correspondence:}

Puja Amatya

MD, Fellowship in Paediatric Critical Care,

Assistant Professor,

Department of Paediatrics,

Patan Academy of Health Sciences, Lagankhel, Lalitpur, Nepal,

Email: pujaamatya@pahs.edu.np

Acknowledgements: The authors are grateful to the late Dr. Neelam Adhikari, who was actively involved in establishing paediatric critical care delivery in Nepal. She participated in all Nepal Paediatric Critical Care Network Working Group (NPCCWG) meetings. Her dedication and encouragement were vital to this group. All the members of Nepal NPCCWG were collaborators in this project including Akhil Tamrakar, Amrit Dhungel, Amrit Ghimire, Anil Shrestha, Anita Lamichhane, Anwesh Bhatta, Anya Sharma, Arun Neopane, Asim Shrestha, Arun Giri, Basant Rai, Biraj Parajuli, Binod Lal Bajracharya, Devendra Shrestha, Ganendra Bhakta Raya, Henis Shakya, Jamun Singh, Janak Koirala, Kalpana Subedi, Nipun Shrestha, Nirajana Kayastha, Om Krishna Pathak, Piyush Kanodia, Prakash Jyoti Pokhrel, Prakash Joshi, Pramila Rajchal, Prithuja Poudel, Pawana Kayastha, Ruby Thakur, Sandeep Singh, Sangita Puri, Santosh Adhikari, Santosh Pathak, Santosh Pokhrel, Shova Shrestha, Srijana Dongol, Sudeep Shrestha, Sumit Agrawal, Raju Kafle, Yograj Sharma, Vidhata KC.

Funding: Nil

Conflict of Interest: None declared

DOI: $10.3126 /$ jnps.v41i2.35055

To cite this article: Amatya P, Shrestha D, Joshi S, Sharma A, Shrestha S, Basnet S. Nepal Paediatric Society Clinical Guidance for Management of Sepsis and Septic Shock in the Paediatric Intensive Care Units in Nepal. J Nepal Paediatr Soc. 2021;41(2): 119-26.

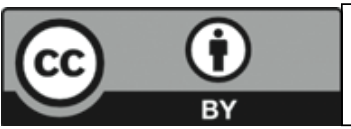

This work is licensed under creative common attribution 3.0 license 


\section{INTRODUCTION}

Transport of critically ill children is a priority in health care that still needs to be addressed in Nepal. ${ }^{1}$ The large burden of sick children, along with relative lack of availability of paediatric intensive care units (PICUs), has led to an increase in referral of patients among health care facilities. ${ }^{2}$ Transport may make a difference in survival of sick children in resource limited countries for this very reason: to reach children to facilities that actually have intensive care services that they may need. ${ }^{3}$ However, sick children are at increased risk of morbidity and mortality during transport. Hence, decision to even transfer needs to be done based on the benefit versus risks associated with transport itself. ${ }^{4}$ Additionally, in Nepal, transport of sick paediatric patients is mostly done in the absence of health care personnel and without any information or documentation for receiving facilities. ${ }^{1,2}$ These inappropriate transports may, in fact, cause deterioration of clinical status and even death, enroute or upon arrival to the receiving hospital / health facility. Studies have shown reduction in morbidity and mortality of critically ill children if transport is carried out correctly, by trained health personnel or specialised teams, after initial stabilisation of the child if needed. ${ }^{5,6}$ This results in less transport-related adverse events, more stable patients on arrival at receiving facility and less inhospital mortality. ${ }^{6-10}$

\section{AIM}

The main aim of this document is to create a consensus guideline and protocol for the safe inter facility transport of critically ill children in Nepal. The protocol will include:

- Emphasis on appropriate emergency treatment and stabilisation of patients prior to transport

- Guideline to anticipate adverse events during transport and their timely management en-route

- Availability of emergency equipment and medications that may be required during transport

- Documentation and communication of required information between referral and receiving facility

\section{METHODOLOGY}

Most of the PICUs in Nepal are run by general paediatricians, as there are only a few paediatric intensive care specialists working in the country. Hence, the paediatricians working in PICUs in different parts of Nepal were contacted and 'Nepal Paediatric Critical Care Working Group' (NPCCWG) was created in 2016. The main objective of this group was to develop evidencebased protocols for PICU, based on locally available resources to improve quality of care of critically ill children in Nepal. As critically ill paediatric patients are at increased risk of morbidity and mortality during transport in a resource limited country alike Nepal, the group agreed that creation of protocol for transport of critically ill children needed to be a priority. Accordingly, the initial draft was developed at the first paediatric critical care workshop of the NPCCWG conducted on $23^{\text {rd }}$ November 2016. After further revisions done at subsequent meetings of the NPCCWG in 2018 and 2019, the final consensus document was presented to the Nepal Paediatric Society (NEPAS). The guideline and protocol for 'Transport of Critically Ill Children in Nepal' was finally endorsed by NEPAS to be published in NEPAS journal for the benefit of critically ill children in Nepal.

\section{Importance of safe transport:}

Critically ill children transported by skilled personnel, with availability of appropriate equipment and medications that may be needed, leads to fewer adverse events and decreased deaths during transport and upon arrival at the health facility.

\section{Types of transport: ${ }^{11}$}

- Intra-hospital transport: Transport of a patient from one site to another within the same hospital for diagnostics or therapeutic interventions.

- Inter-hospital transport: Transport of a patient from one hospital to another hospital.

Common indications for transport: ${ }^{12}$

- Unavailability of beds or ventilator

- Unavailability to treat critically ill patients within the facility

- Diagnostic and therapeutic interventions

- On request of the family 


\section{Components of transport:}

When potential transfer is identified, both providers and patients and their parents / legal guardians need to be prepared for referral to another health facility.

1. Communication with parents: This is one of the important aspects of transport of critically ill children. Parents must be counselled regarding the reason for transferring their child to another health facility, and ensure that they agree to this.

2. Communication with referral facility: Effective communication with the receiving facility is one of the most important factors to determine successful transport. It is the responsibility of the referring facility to communicate with the receiving facility to ascertain availability of bed and / or ventilator prior to transport. The receiving facility should be provided with a brief summary of patient's condition, all imaging and laboratory work done, treatment administered and the reason for referral.

3. Means of transport: The appropriate means of transport should be identified (air transport versus ambulance by road) and arranged after discussion with the family. Ensure that the ambulance brings the transfer team back to the referring hospital.

4. Patient preparation: As soon as transfer is planned, patient should be prepared according to the transfer checklist provided below (APPENDIX I).

5. Stabilisation of child before transport: This is a very crucial part for management of sick children. It is the responsibility of the referring facility to stabilise the child pre-transport. Transporting a sick child without stabilisation will lead to serious adverse events, including death.

6. Equipment preparation: As soon as transport is planned, equipment needs to be checked. It's always better to keep equipment ready in a bag or box that is used during transfer of sick children. Oxygen cylinder needs to be checked. It is advised to carry one extra cylinder in the ambulance. Table 1 provides the details of equipment lists that should be used in transport of critically ill children.

7. Personnel and training: During transfer, sick children should be accompanied by health personnel. If the child is intubated, he / she should be accompanied by two health personnel and at least one should know how to intubate the child. Ideally, transport personnel should be trained on Basic Life Support (BLS), Paediatric Advance Life Support (PALS), Neonatal Resuscitation Program (NRP). It is always better to send health personnel who have some experience of working in PICU for transport of a critical child.

8. Documents: Patient should be transferred with a copy of transfer summary, radiographic studies, laboratory investigation results, medication list and referral slip as provided below (APPENDIX II).

9. Patient assessment before leaving the referral unit: Patient should be assessed including general physical examination, vital signs, along with systemic evaluation before transporting the child. Transport personnel should evaluate whether the child is stable enough to be transferred. If the child is not stable, he / she should be transferred only after stabilisation.

10. Duration of transport: The start time of transport from referral facility and time of arrival at receiving facility should be noted. Any adverse events during the transport should be noted along with any intervention performed.

11. Patient assessment at arrival at the receiving facility: Patient should be assessed by a health personnel including vital signs check on arrival to the receiving facility. After assessment and documentation of the findings, the patient should be handed over to receiving unit with all the documents brought along.

12. Returning back to hospital: Once transport process is completed; transport personnel should return with the equipment and transfer checklist back to the referring facility.

\section{CONCLUSIONS}

This clinical guideline for management of sepsis and septic shock in children in Nepal is a consensus document, keeping in view the resources available, created by paediatricians working in various PICUs in the country and based / adapted from the Surviving Sepsis Campaign 2020. This is an 
attempt to decrease variability in critical care delivery and standardise care by providing a guide to providers. Use and efficacy of this guideline will be monitored after dissemination and implementation.

Table 1. Equipment list

\section{Equipment lists}

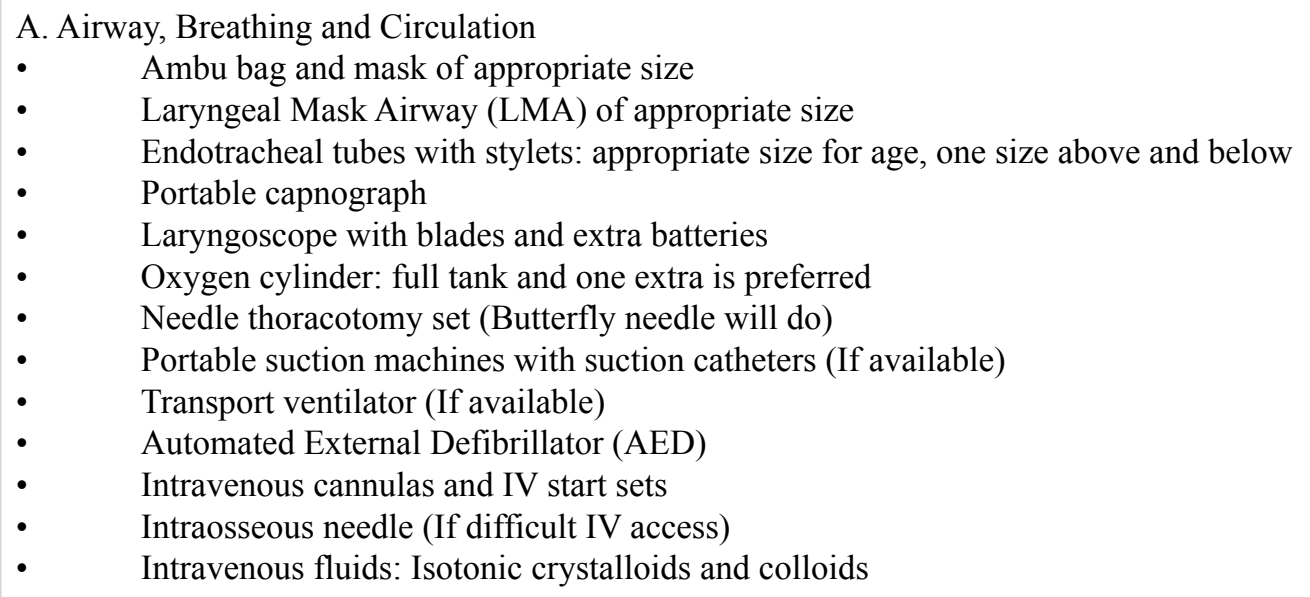

B. Medications

- Adrenaline

- Atropine

- $\quad$ Normal saline

- $10 \%$ Dextrose

- $10 \%$ Calcium gluconate

- Morphine

- $\quad$ Midazolam

- $\quad$ Anticonvulsants (If seizure)

- Inotropes (If shock)

- Mannitol or 3\% saline (If raised intracranial pressure)

C. Monitoring

- $\quad$ Pulse oximeter (Ensure it is charged)

- Thermometer

- $\quad$ Stethoscopes

- Glucometer

- $\quad$ Blood pressure cuff (Appropriate size)

- $\quad$ Syringe pumps (If available) or intravenous set

- $\quad$ Monitor with ECG leads (If available) 


\title{
Appendix I
}

\section{Transfer Checklist (Referring Hospital to complete)}

\section{Doctor / Medical staff accompanying the patient needs to complete this}

\author{
Name: \\ Age: \\ Sex: \\ Hospital no: \\ Date of Transfer: \\ Time of transfer: \\ Transfer from: \\ Transfer to:
}

\begin{tabular}{|l|l|}
\hline 1. WHEN POTENTIAL TRANSFER IS IDENTIFIED & $\begin{array}{c}\text { Check [ }] \text { ] } \\
\text { [N/A not available } \\
\text { or not applicable] }\end{array}$ \\
\hline Identify the problem and the reason for transfer & \\
\hline Inform the senior doctor on call & \\
\hline Inform parents & \\
\hline Ensure that the problem of the patient is communicated to the receiving unit \\
\hline Ensure that the receiving unit is ready to receive and bed is available \\
\hline Identify the transfer team & \\
\hline Evaluate urgency of transfer & \\
\hline Prepare equipment for transfer & \\
\hline Book ambulance with oxygen and ensure that the ambulance will take the transfer team back to hospital & \\
\hline
\end{tabular}

\section{PATIENT PREPARATION}

Patient must have a definitive airway. If in doubt, intubate electively.

Secure endotracheal tube

Ensure normal blood glucose

Tape the eyes of the patient if sedated

If history of trauma, immobilize the cervical spine

Chest drains must never be clamped. Use one way valve drainage bag instead of underwater seal bottles if possible

At least two reliable intravenous accesses should be obtained

For short transfers and or for older children, maintenance fluid may sometimes be omitted but must be carried

Neonates: intravenous fluid should be continued (via a syringe pump, if available)

Sedation and paralyzing agents can be given by bolus injections

Ensure patient is wrapped properly to prevent hypothermia

\section{EQUIPMENT PREPARATION}

Resuscitation Box : Endotracheal tube $\square$, stylet $\square$, Ambubag $\square$, laryngoscope $\square$, with blades and extra batteries $\square$, syringes $\square$, Adrenaline $\square$, Atropine $\square$, Normal saline $\square, 10 \%$ Dextrose $\square$, Morphine $\square$,

Midazolam $\square$, Calcium gluconate $\square$, thermometer $\square$, glucometer $\square$, BP instrument[app. cuff] $\square$

Transfer cot for small babies

Portable SPO2 machine (ensure that it is charged fully)

Portable O2: keep extra cylinder

Patient's transfer notes $\square \quad$ X-rays $\square \quad$ investigation reports $\square$

Check $[\sqrt{ }]$

available items

Check $[\sqrt{ }]$

available items

- $\quad$ Ensure the transfer team has a mobile phone, the receiving unit's contact no and the contact no of

a senior doctor on call from the referring unit

- Money for emergency

- Confirm route to receiving unit

Reason for transfer: 
Time of leaving the referral unit:

Vital signs on leaving the referral unit:

- Temperature:

- Heart Rate:

- Respiratory Rate:

- $\quad \mathrm{CRT} / \mathrm{BP}$ :

- $\quad \mathrm{SPO}_{2}$ :

- Blood Glucose:

Time of arrival at the receiving unit:

Assessment of child on arrival at the receiving unit:

- Temperature:

- Heart Rate:

- Respiratory Rate:

- $\mathrm{CRT} / \mathrm{BP}$ :

- $\mathrm{SPO}_{2}$ :

- Blood Glucose:

- Airway status:

- Breathing:

- Circulation status:

- Disability: Neurological status: AVPU

Pupils (size and reaction):

- Nutrition: Enteral or parenteral:

- Sedation status (if yes): medicines and dose:

Adverse events during transfer if any:

Receiving unit / Doctor's Name and Signature:

Name and signature of the transferring Doctor: 


\section{APPENDIX II (Referring Hospital to complete)}

\section{Critically ill Child Referral Slip}

Code

Name:

Age:

Sex:

Provisional Diagnosis:

Name of referring hospital:

Phone no:

Name of receiving hospital:

Phone no:

Date and time of referral:

Estimated duration of travel:

Reason for referral:

During transport accompanied by: Doctor / Nurse / Paramedics / Other Health worker / Parents / guardians

Brief History and Hospital course (Relevant only): Or a discharge summary

Vitals at the time of referral: Temp.: $\quad$ PR: $\quad$ BP: $\quad$ CRT: $\quad$ RR: $\quad$ SpO2: $\quad$ Blood Sugar:

Level of Consciousness: AVPU or GCS

If on ventilator, ventilator settings:

PIP: $\quad$ Rate: $\quad \mathrm{FiO}_{2}: \quad \mathrm{TV}: \quad$ Minute ventilation:

Blood Gas: ABG: $\square \quad$ CBG: $\square \quad$ VBG: $\square$

$\mathrm{pH}: \quad \mathrm{pO}_{2}: \quad \mathrm{HCO}_{3}: \quad \mathrm{pCO}_{2}:$ Base excess:

Laboratory reports (Relevant only):

Treatment received:

Consultant / Resident / House officer 


\section{REFERENCES}

1. Basnet S, Shrestha S, Ghimire A, Timila D, Gurung J, Karki U, et al. Development of a PICU in Nepal: the experience of the first year. Pediatr Crit Care Med. 2014;15:314-20. DOI: https://doi.org/10.1097/pcc. 0000000000000201

2. Khanal A, Sharma A, Basnet S. Current State of Paediatric Intensive Care and High Dependency Care in Nepal. Pediatr Crit Care Med. 2016;17:1032-40. DOI: https://doi.org/10.1097/pcc.0000000000000938

3. Prabhudesai S, Kasala M, Manwani N, Krupanandan R, Ramachandran B. Transport related adverse events in critically-ill children: the role of a dedicated transport team. Indian Pediatr. 2017;54:942-5. DOI: https://doi.org/ 10.1007/s13312-017-1187-y

4. Bruzzini CDB, Mysore MR, Conway EE, Pringle EJ, Gayle MO, Serrao KL, et al. Transport of the critically ill child. In: Madden MA, editor. Pediatric Fundamental Critical Care Support. $2^{\text {nd }}$ ed. USA: Society of Critical Care Medicine; 2013. p 14(1)-14(16)

5. Britto J, Nadel S, Maconochie I, Levin M, Habibi P. Morbidity and severity of illness during interhospital transfer: specialized pediatric retrieval team. Br Med J. 1995;311:836-9. DOI: https://doi.org/10.1136/bmj.311.7009.836

6. Vos GD, Nissen AC, Nieman FH, Meurs MM, van Waardenburg DA, Ramsay G, et al. Comparison of interhospital pediatric intensive care transport accompanied by a referring specialist or a specialist retrieval team. Intensive Care Med. 2004;30:302-8. DOI: https://doi.org/10.1007/s00134-003-2066-7

7. Chance GW, Matthew JD, Gash J, Williams G, Cunningham K. Neonatal transport: A controlled study of skilled assistance. Mortality and morbidity of neonates less than $1.5 \mathrm{~kg}$ birth weight. J Pediatr. 1978;93:662-6. DOI: https:// doi.org/10.1016/S0022-3476(78)80913-5

8. Edge WE, Kanter RK, Weigle CG, Walsh RF. Reduction of morbidity in inter-hospital transport by specialised pediatric staff. Crit Care Med. 1994;22:1186-91. DOI: https://doi.org/10.1097/00003246-199407000-00023

9. Macnab AJ. Optimal escort for interhospital transport of paediatric emergencies. J Trauma. 1991;31:205-9. DOI: https://doi.org/10.1097/00005373-199131020-00009

10. Hatherill M, Waggie Z, Reynolds L, Argent A. Transport of critically ill children in a resource-limited setting. Intensive Care Med. 2003;29:1547-54. DOI: https://doi.org/10.1007/s00134-003-1888-7

11. Khilnani P, Chhabra R. Transport of Critically ill Children: How to Utilize Resources in the Developing World. Ind J Pediatr. 2008;75:591-8. DOI: https://doi.org/10.1007/s12098-008-0115-5

12. Kamath SR, Gandhi D. Transport of Sick Children. In Gogoi N, editor. Pediatric Critical Care Manual. New Delhi: Wolters Kluwer Health (India) Pvt. Ltd.; 2018. p 18-23. 